\title{
The Ser19Stop single nucleotide polymorphism (SNP) of human PHYHIPL affects the cerebellum in mice
}

\author{
Hisako Sugimoto ${ }^{1}$, Takuro Horii ${ }^{2}$, Jun-Na Hirota ${ }^{3}$, Yoshitake Sano ${ }^{3}$, Yo Shinoda ${ }^{4}$, Ayumu Konno ${ }^{5}$, Hirokazu Hirai ${ }^{5}$, \\ Yasuki Ishizaki ${ }^{6}$, Hajime Hirase ${ }^{7}$, Izuho Hatada ${ }^{2}$, Teiichi Furuichi ${ }^{3}$ and Tetsushi Sadakata ${ }^{1 *}$ (D)
}

\begin{abstract}
The HapMap Project is a major international research effort to construct a resource to facilitate the discovery of relationships between human genetic variations and health and disease. The Ser19Stop single nucleotide polymorphism (SNP) of human phytanoyl-CoA hydroxylase-interacting protein-like (PHYHIPL) gene was detected in HapMap project and registered in the dbSNP. PHYHIPL gene expression is altered in global ischemia and glioblastoma multiforme. However, the function of PHYHIPL is unknown. We generated PHYHIPL Ser19Stop knock-in mice and found that PHYHIPL impacts the morphology of cerebellar Purkinje cells (PCs), the innervation of climbing fibers to PCs, the inhibitory inputs to PCs from molecular layer interneurons, and motor learning ability. Thus, the Ser19Stop SNP of the PHYHIPL gene may be associated with cerebellum-related diseases.
\end{abstract}

Keywords: PHYHIPL, PHYHIP, dbSNP, HapMap Project, Cerebellum, Purkinje cell

\section{Introduction}

PHYHIP-like (PHYHIPL) is a paralog of the phytanoylCoA hydroxylase-interacting protein (PHYHIP). Hereafter, we abbreviate PHYHIPL as PHY2 and the original PHYHIP as PHY1. Kim and colleagues first cloned PHY1, which showed a brain-specific expression pattern [1]. They also reported that PHY1 interacts with the Refsum disease gene product phytanoyl-CoA alpha-hydroxylase (PAHX) [1] and brain-specific angiogenesis inhibitor 1 (BAI1) [2]. Another group reported that PHY1 interacts with a dual-specificity tyrosine-phosphorylated and regulated kinase 1A (DYRK1A), which is located in a Down syndrome critical region [3]. Besides, visual stimulation is essential to maintain PHY1 expression in the retina [4]. On the other hand, the gene expression of PHY2 is

\footnotetext{
*Correspondence: sadakata-1024@umin.ac.jp

1 Education and Research Support Center, Gunma University Graduate School of Medicine, 3-39-22 Showa-machi, Maebashi, Gunma 371-8511, Japan

Full list of author information is available at the end of the article
}

altered in global ischemia [5] and glioblastoma multiforme [6], but its function remains unknown.

The International HapMap Consortium has developed the HapMap, a resource that describes the common patterns of human genetic variation. HapMap data are deposited in the Single-Nucleotide Polymorphism database (dbSNP), a variation database at the National Center for Biotechnology Information (NCBI). The Ser19Stop SNP of the human PHY2 gene was identified by the HapMap project and registered in the dbSNP (Database ID: rs7907875). The frequency of the Ser19Stop SNP of human PHY2 in the Japanese population is high (2.3\%). However, all Ser19Stop SNP are registered as heterozygote and the HapMap project identified no homozygote. There is a $0.05 \%$ chance of finding heterozygotes in the Japanese population. Homozygotes may suffer from some disease, which would explain why they have been omitted from the collection of DNA. Therefore, we generated homozygous Ser19Stop PHY2 knock-in mice and original author(s) and the source, provide a link to the Creative Commons licence, and indicate if changes were made. The images or other third party material in this article are included in the article's Creative Commons licence, unless indicated otherwise in a credit line to the material. If material is not included in the article's Creative Commons licence and your intended use is not permitted by statutory regulation or exceeds the permitted use, you will need to obtain permission directly from the copyright holder. To view a copy of this licence, visit http://creativecommons.org/licenses/by/4.0/. The Creative Commons Public Domain Dedication waiver (http://creativeco mmons.org/publicdomain/zero/1.0/) applies to the data made available in this article, unless otherwise stated in a credit line to the data. 
analyzed their phenotypes to examine the association between some human diseases and the Ser19Stop SNP.

\section{Results and discussion}

The mouse PHY2 and PHY1 proteins share $75 \%$ aminoacid sequence identity (Fig. 1a). PHY2 has structural features similar to the Fibronectin type-III domain, which may mediate specific protein-protein interactions in both intracellular and extracellular compartments
(Fig. 1a). The RT-PCR analysis showed a high PHY2 mRNA expression in the brain and testis (Fig. 1b), indicating that PHY1 and PHY2 have a similar tissue distribution [1].

The Ser19Stop SNP of human PHY2 was registered in the dbSNP (Fig. 1c). We found this SNP manually among the SNPs corresponding to the following search criteria: stop gained [function class] AND by frequency [validation status] AND by cluster [validation status] AND

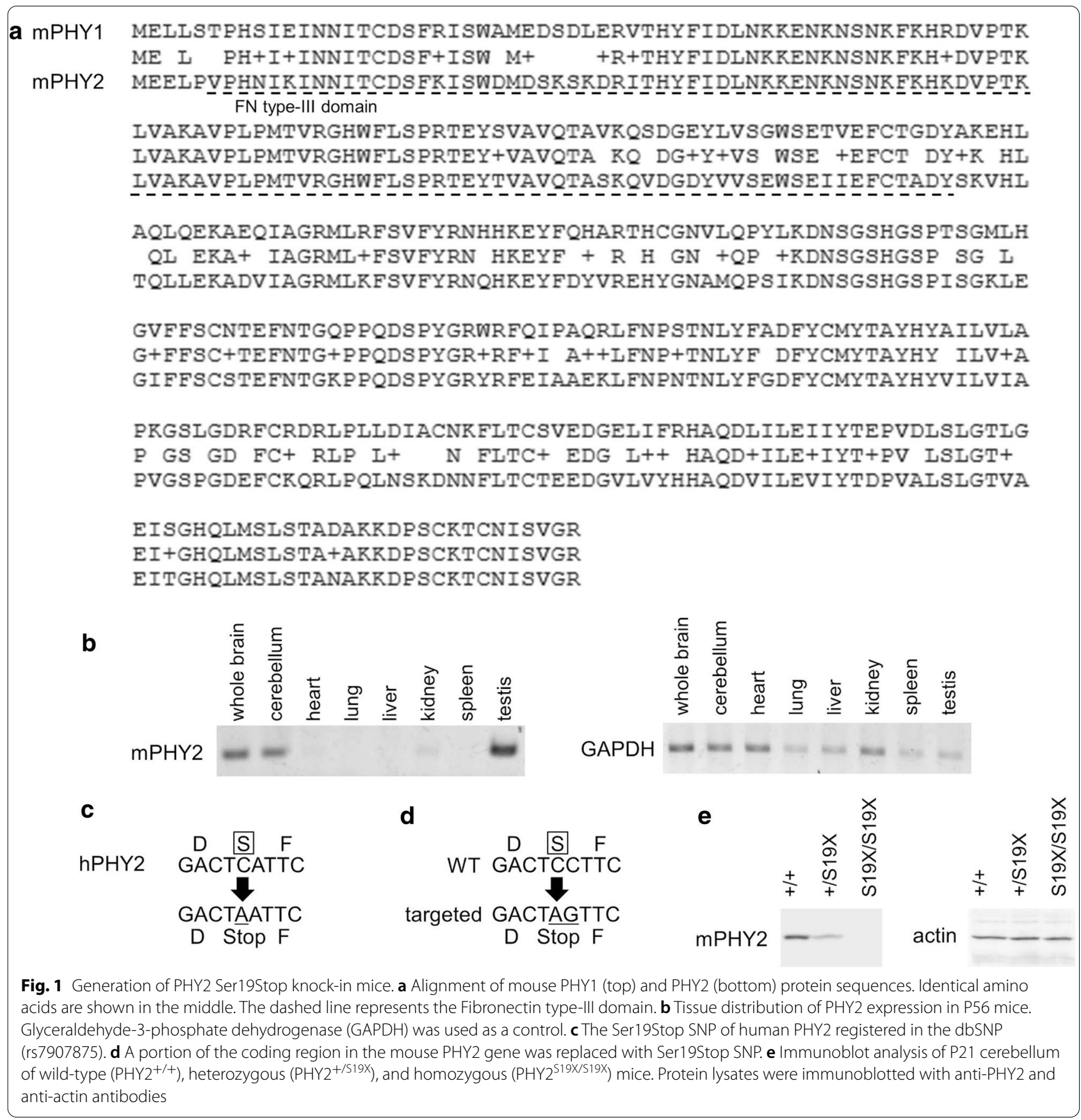


Homo sapiens [organism]. The frequency of Ser19Stop in Japanese and American populations is $2.3 \%$ and $0.9 \%$, respectively. All Ser19Stop SNPs registered in the HapMap project are heterozygotes. The mouse and human PHY2 are highly homologous and both have a serine in position 19 (Additional File. 1: Fig. S1). Therefore, we inserted the Ser19Stop (S19X) mutation in mouse PHY2 by CRISPR/Cas9-mediated gene knock-in strategy (Fig. 1d, Additional File. 1: Fig. S1). We verified the S19X knock-in by sequencing and Western blotting (Fig. 1e).

The expression of PHY1 is brain-specific [1]. In situ hybridization analyses showed that cerebellar granule cells, hippocampal neurons, and cerebral neurons strongly expressed PHY1 mRNA as previously reported (Fig. 2a, b) [1]. Moreover, we detected low levels of PHY1 mRNA in the striatum and nucleus accumbens (Fig. 2b).

On the contrary, PHY2 was distributed over the whole brain area (Fig. 2c, d). The hippocampal granule and pyramidal cells (Fig. 2c, d), neocortical cells located in the deep layers (Fig. 2c, d), and cerebellar Purkinje cells
(Fig. 2g, h) highly expressed PHY2 mRNA. In the cerebellum, the stellate and Golgi cells also weakly expressed PHY2 mRNA (Fig. 2h).

Apart from the cerebellum, the brain structure and cytoarchitecture of the PHY2 mutant homozygotes $\left(\mathrm{PHY} 2^{\mathrm{S} 19 \mathrm{X} / \mathrm{S} 19 \mathrm{X}}\right.$ ) were indistinguishable from those seen in wild-type littermates. In the cerebellum, the PHY2 ${ }^{\text {S19X/S19X }}$ Purkinje cells (PCs) had significantly lower cell density than wild-type PCs (Fig. 3a-c). Besides, the PHY2 ${ }^{\text {S19X/S19X }}$ PC soma was smaller than that of wild-type (Fig. 3d). PCs are pear-shaped neurons and their longitudinal diameter is frequently bigger than the transversal diameter (Fig. 3a). Interestingly, the $\mathrm{PHY} 2^{\mathrm{S} 19 \mathrm{X} / \mathrm{S} 19 \mathrm{X}} \mathrm{PCs}$ were round-shaped and their soma had a smaller height/width ratio than that of wild-type PC soma (Fig. $3 \mathrm{e}-\mathrm{g}$ ). Postmortem neuropathology studies report that a majority of cerebellar specimens from persons diagnosed with autism spectrum disorders have fewer and smaller PCs [7]. Having few and small PCs may reduce the directional
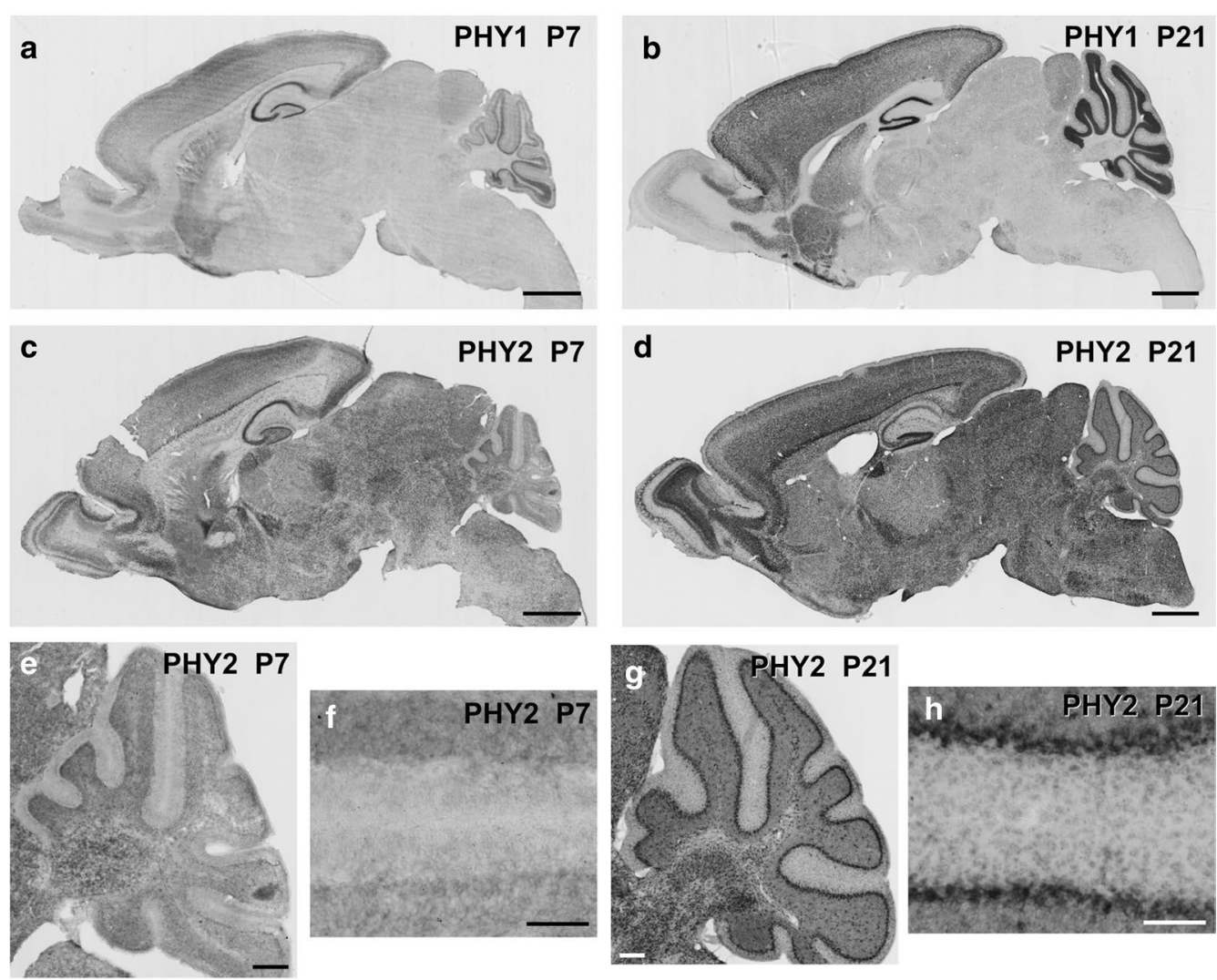

Fig. 2 Expression of PHY1 and PHY2 mRNA in mouse brains. $\mathbf{a}$, b In situ hybridization analysis of PHY1 mRNA distribution in P7 (a) and P21 (b) sagittal brain sections. Scale bars: 1 mm. c, d In situ hybridization analysis of PHY2 mRNA distribution in P7 (c) and P21 (d) sagittal brain sections. Scale bars: 1 mm. e In situ hybridization analysis of PHY2 mRNA distribution in P7 sagittal brain sections in the cerebellum. Scale bars: $200 \mu$ m. f In situ hybridization analysis of PHY2 mRNA distribution in P7 sagittal brain sections in the cerebellar cortex. Scale bars: $100 \mu \mathrm{m}$. $\mathbf{g} \mathrm{In}$ situ hybridization analysis of PHY2 mRNA distribution in P21 sagittal brain sections in the cerebellum. Scale bars: $200 \mu \mathrm{m}$. $\mathbf{h}$ In situ hybridization analysis of PHY2 mRNA distribution in P21 sagittal brain sections in the cerebellar cortex. Scale bars: $100 \mu \mathrm{m}$ 

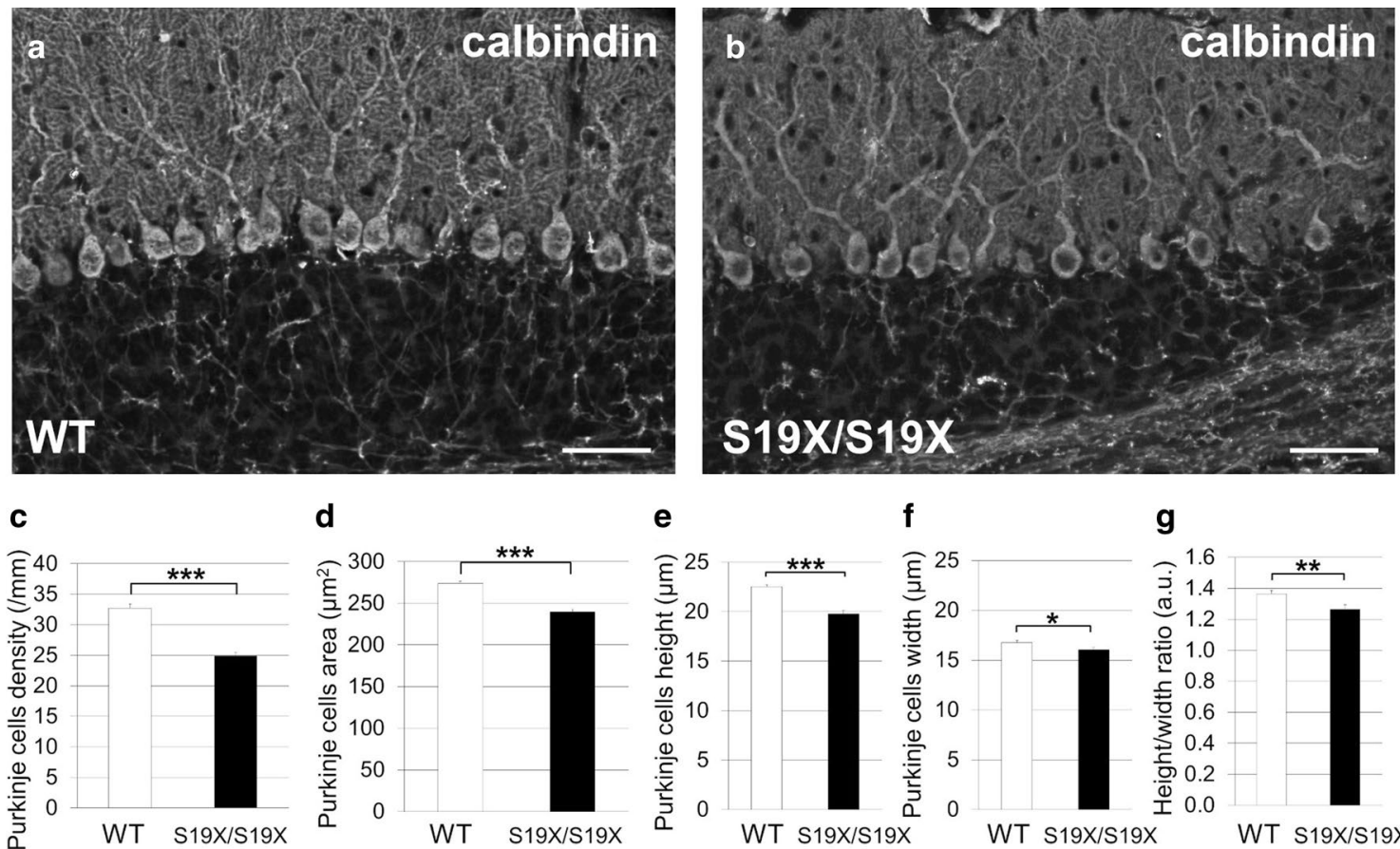

f

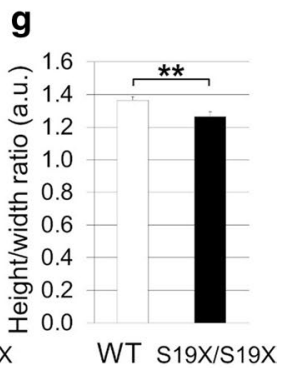

Fig. 3 Abnormal PC morphology in PHY2 ${ }^{\text {S19X/S19X }}$ cerebellum. a, b Sagittal sections of P21 wild-type (WT) (a) and PHY2 ${ }^{\text {S19X/S19X }}$ (S19X/S19X) (b) mouse cerebella immunolabeled with an anti-calbindin antibody. Scale bars: $50 \mu \mathrm{m}$. c Cell densities of calbindin-positive PCs for WT (white, $n=40$ ) and PHY2 ${ }^{\text {S19X/S19X }}$ (black, $n=71$ ) mice at P21. d PC soma size for WT (white, $n=315$ ) and PHY2 ${ }^{\text {S19X/s19X }}$ (black, $\left.n=289\right)$ mice at P21. e Longitudinal diameter (height) of the PC soma for WT (white, $n=124$ ) and PHY2 ${ }^{\text {S19X/S19X }}$ (black, $n=106$ ) mice at P21. $\mathbf{f}$ Transversal diameter (width) of the PC soma for WT (white, $n=124$ ) and PHY $2^{519 X / 519 X}$ (black, $n=106$ ) mice at P21. $P=0.0314$, Student's $t$ test. $\mathbf{g}$ Height/width ratio of the PC soma for WT (white, $n=124$ ) and PHY2 ${ }^{\text {S19X/S19X }}$ (black, $n=106$ ) mice at P21. $P=0.00424$, Student's $t$ test. The error bars indicate the s.e.m. ${ }^{*} P<0.05$; ${ }^{*} P<0.01$; *** $P<0.001$, Student's $t$ test

coherence and detection of streamlines connecting the cerebellar cortex with the dentate nucleus [8].

In the developing cerebellum, climbing fibers (CFs) surround the basal part of monolayered PC somata and establish synaptic contacts with perisomatic protrusions and thorns. Perisomatic CF synapses then progressively translocate to growing $\mathrm{PC}$ dendrites between postnatal week one to three in the mouse cerebellum [9]. When the number of perisomatic CF synapses decreases, the height of the CF projection in the molecular layer (ML) dramatically increases. Dendritic translocation of CFs must be an activity-dependent process, as administering tetrodotoxin or AMPA receptor blockers atrophy CF innervation in adult rats and mice [10-12]. The dendritic translocation is evaluated as the height of CF projection relative to the thickness of the ML [9]. The thickness of the ML was slightly smaller in the PHY2 ${ }^{\mathrm{S} 19 \mathrm{X} / \mathrm{S} 19 \mathrm{X}}$ mouse cerebellum than in the wild-type cerebellum (Fig. $4 \mathrm{a}-\mathrm{c}$ ). Moreover, the relative height of CF to ML was smaller in the PHY2 ${ }^{\mathrm{S} 19 \mathrm{X} /}$ S19X cerebellum than in the wild-type cerebellum (Fig. 4d). The wild-type and PHY2 ${ }^{\text {S19X/S19X }}$ cerebellum had the same vGluT2 puncta density (Fig. 4e). These results indicate that decreased PC activity occurs in the PHY2 ${ }^{\text {S19X/S19X }}$ mouse cerebellum.

PCs receive two excitatory inputs, from parallel fibers (axons of the granule cells) and climbing fibers. They receive inhibitory inputs from two groups of ML interneurons, the basket cells and stellate cells. The spatiotemporal patterns of PC action potentials, which are triggered by the excitatory inputs, are fine-tuned by the inhibitory inputs from ML interneurons [13, 14]. To clarify the effects of PHY2 on the formation of ML interneuron-PC synapses, we first evaluated the density of VGAT-positive inhibitory terminals. PHY2 ${ }^{\text {S19X/S19X }}$ ML had a smaller density of VGAT puncta than wildtype ML (Fig. $5 \mathrm{a}-\mathrm{c}$ ). The cell density in $\mathrm{PHY}^{\mathrm{S} 19 \mathrm{X} / \mathrm{S} 19 \mathrm{X}}$ and wild-type ML was the same (Fig. 5d). The decrease in VGAT puncta in Fig. 5 may be due to homeostatic plasticity, a process by which neurons adapt to the overall network activity to maintain their firing rates [15]. In dissociated primary cultures of rat neocortex, decreased neuronal activity leads to a decrease in VGAT [16]. Similarly, decreased activity of Purkinje cells may lead to a decrease in VGAT expression in surrounding interneurons due to homeostatic plasticity (Fig. 5c). To conclude 

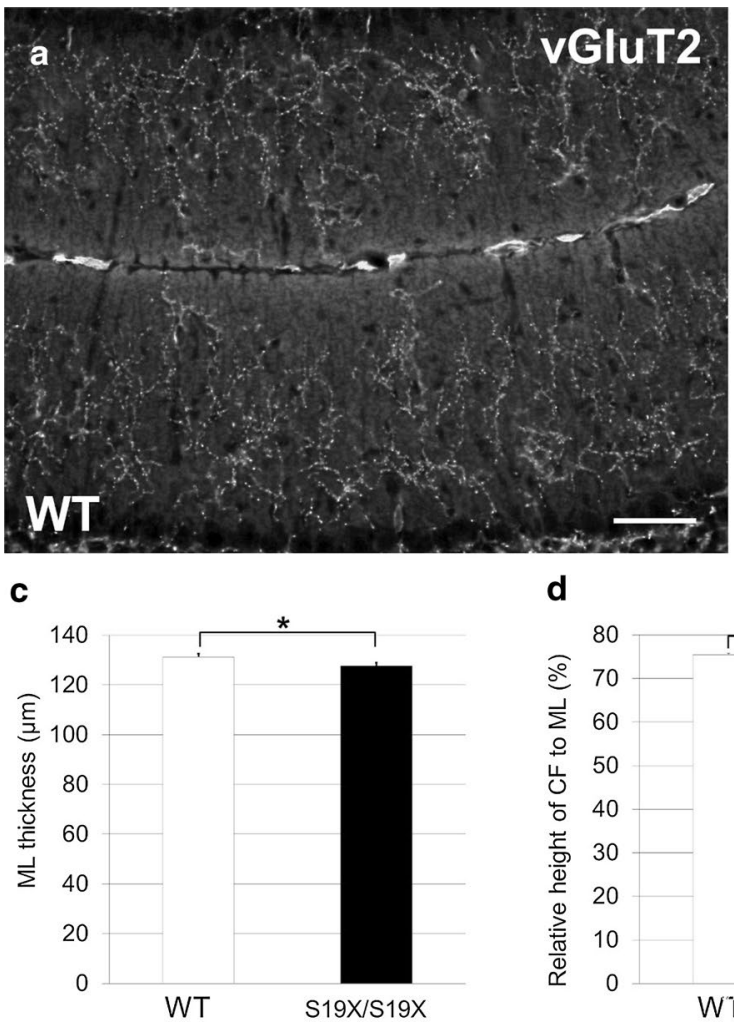

d

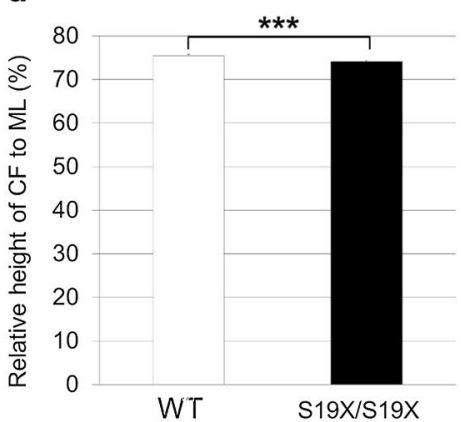

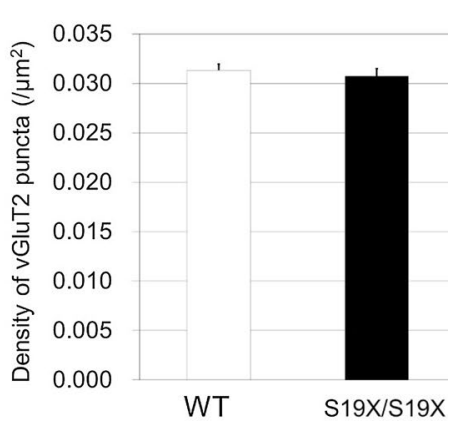

Fig. 4 Decreased CF height relative to $M L$ in the PHY2 ${ }^{S 19 X / S 19 X}$ cerebellum. a, b Sagittal sections of P21 WT (a) and PHY2 $2^{S 19 X / S 19 X}(\mathbf{b})$ mice cerebella immunolabeled with an anti-vGluT2 antibody. Scale bars: $50 \mu \mathrm{m}$. c ML thickness in WT (white, $n=340)$ and PHY2 ${ }^{519 x / 519 x}$ (black, $\left.n=340\right)$ mice at $P 21 . P=0.0493$, Student's $t$ test. $\mathbf{d}$ CF height relative to ML in WT (white, $n=340$ ) and PHY2 ${ }^{\text {S19X/S19X }}($ black, $n=340)$ mice at P21. e Density of vGluT2 puncta in the ML of WT (white, $n=340)$ and PHY2 ${ }^{S 19 X / 519 X}($ black, $n=340)$ mice at P21. The error bars indicate the s.e.m. ${ }^{*} P<0.05$; ${ }^{* * *} P<0.001$, Student's $t$ test

the association between our immunohistochemical results and Purkinje cell activity, the physiological activity of Purkinje cells in the PHY2 $2^{\mathrm{S} 19 \mathrm{X} / \mathrm{S} 19 \mathrm{X}}$ cerebellum remains to be elucidated.

Since, in home cages, the PHY2 $2^{\mathrm{S} 19 \mathrm{X} / \mathrm{S} 19 \mathrm{X}}$ mice had a behavior indistinguishable from that of their wild-type littermates, we analyzed their motor ability more carefully using a rotarod apparatus. Mice of both genotypes learned the task, and the time on the rod gradually increased (Fig. 5e). We monitored the motor learning ability during the test periods. The motor coordination of $\mathrm{PHY} 2^{\mathrm{S} 19 \mathrm{X} / \mathrm{S} 19 \mathrm{X}}$ mice was significantly poorer on the fourth testing day $[F(1,51)=4.70, P=0.0334]$. These results suggest that $\mathrm{PHY} 2^{\mathrm{S} 19 \mathrm{X} / \mathrm{S} 19 \mathrm{X}}$ mice have impaired motor coordination.

PHY1 interacts with the Refsum disease gene product PAHX [1], BAI1 [2], and DYRK1A [3]. Therefore, to examine the protein function of PHY2, we measured its binding activity to these three candidates. Figure 6 shows that that none of them bound to PHY2.

The Ser19Stop mouse had morphologically altered cerebellar PCs, abnormal projection of CFs to PC dendrites, decreased formation of ML interneuronPC synapses, and impaired motor coordination. These results show that Ser19Stop SNP may be associated with cerebellum-related diseases. Thus far, we could not elucidate the PHY2 protein function. We used the Prosite domain database to identify the fibronectin type III domain in PHY2 [17]. The fibronectin type III superfamily has an Ig-like $\beta$-sandwich fold [18]. The fibronectin type III domain is a homophilic binding site involved in fibronectin matrix assembly. The interaction between type III and type I domains is critical for the homophilic binding of fibronectin [19]. Therefore, PHY2 may interact with other proteins through its fibronectin type III domain and have distinct functions from PHY1. Thus far, PHY2 binding proteins have been identified by recombinant PHY2 expression in some neuronal cell lines (data not shown). The association between PHY2 SNPs and human diseases remains unelucidated and requires detailed analyses. 


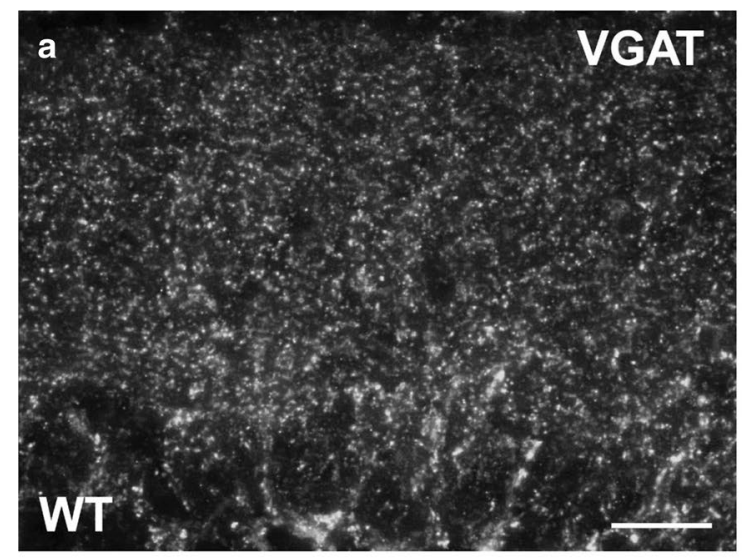

C

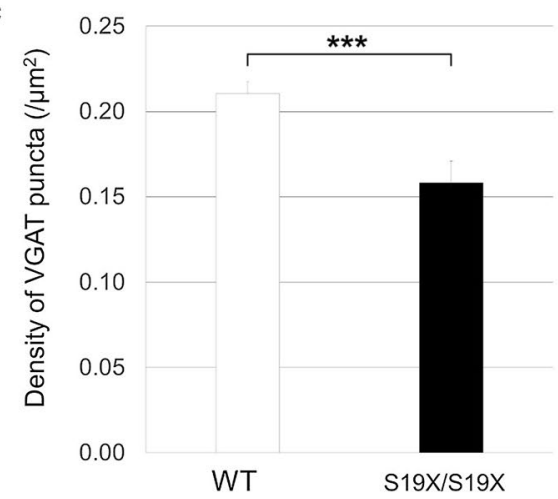

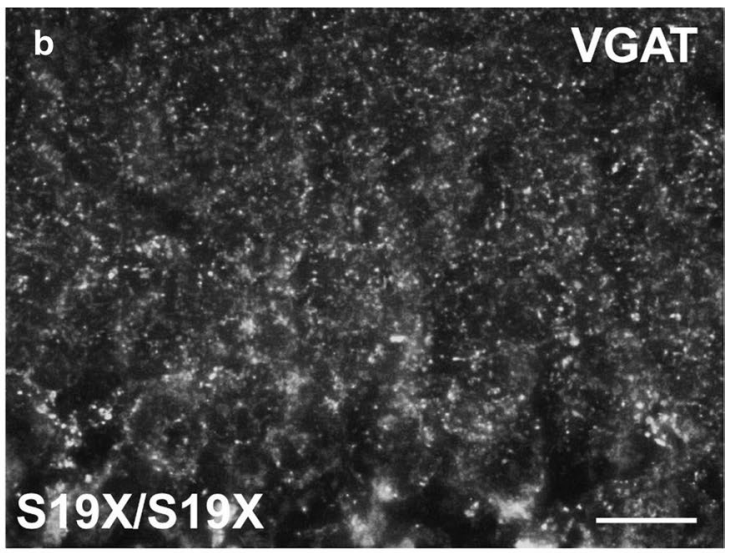

d

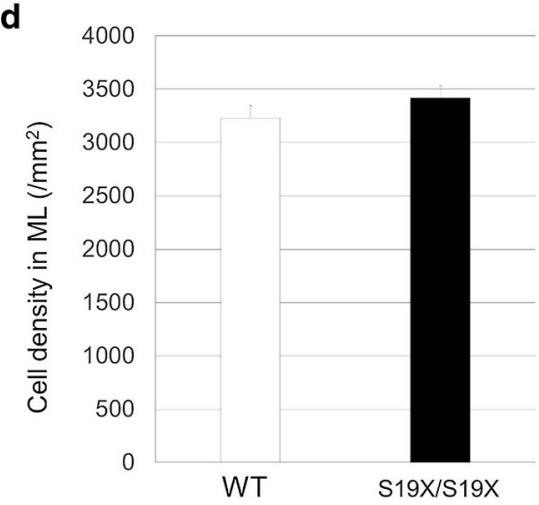

e

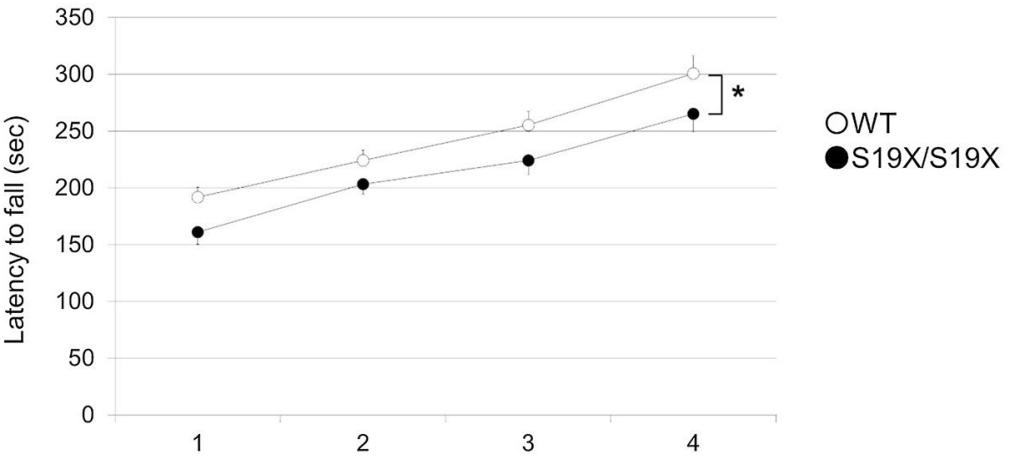

Fig. 5 Decreased density of VGAT puncta in the ML of the PHY2 ${ }^{\mathrm{S19X/S19X}}$ cerebellum and impairment of motor coordination in PHY $2^{\mathrm{S19X} / \mathrm{S19X}}$ mice. a, b Sagittal sections of P21 WT (a) and PHY2 $2^{\text {S19X/S19X }}(\mathbf{b})$ mice cerebella immunolabeled with an anti-VGAT antibody. Scale bars: $20 \mu \mathrm{m}$. c Density of VGAT puncta in the ML of WT (white, $n=31$ ) and PHY2 ${ }^{\text {S19X/S19X }}$ (black, $n=30$ ) mice at P21. d Density of DAPI positive cells in the ML of WT (white, $n=38$ ) and PHY2 ${ }^{\text {S19X/S19X }}$ (black, $n=48$ ) mice at P21. e Fall latency in the rotarod test for WT (white, $n=11$ ) and PHY2 ${ }^{\text {S19X/S19X }}$ (black, $\left.n=8\right)$ mice at postnatal week 10-11. Data for the 4 days of evaluation is shown. ${ }^{*} P=0.0334$, repeated measures ANOVA. The error bars indicate the s.e.m. ${ }^{* * *} P<0.001$, Student's $t$ test. ${ }^{*} P<0.05$, repeated measures ANOVA

\section{Materials and methods}

\section{Generation of PHYHIPL Ser19Stop knock-in mice}

We generated the PHYHIPL Ser19Stop knock-in mice using the electroporation method according to our previous report with some modifications [20]. In this study, we used synthetic crRNA (Alt-R ${ }^{\circledR}$ CRISPR-Cas9 crRNA; IDT, Coralville, IA, USA) and tracrRNA (Alt- ${ }^{\circledR}$ CRISPR-Cas9
tracrRNA, IDT) instead of single guide RNA (sgRNA). We also designed the corresponding donor singlestranded oligodeoxynucleotides (ssODNs) with $5^{\prime}$ - and 3'-homology arms. Target sequence: CATATCCCATGA GATCTTGAAGG. Equal volumes of crRNA and tracrRNA were combined in a duplex buffer (IDT) in a thermal cycler at $95^{\circ} \mathrm{C}$ for 5 min following the manufacturer's 


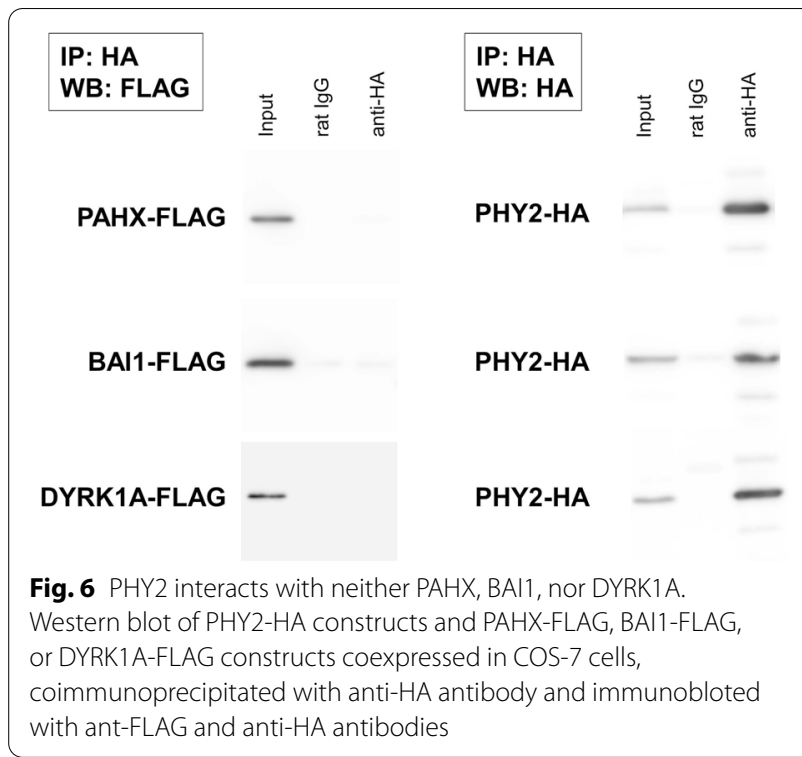

protocol. crRNA/tracrRNA $(3 \mu \mathrm{M})$, recombinant Cas9 protein $\left(100 \mathrm{ng} / \mu \mathrm{L} ;\right.$ GeneArt Platinum ${ }^{\mathrm{TM}}$ Cas 9 Nuclease, Thermo Fisher Scientific, Waltham, MA, USA) and $\operatorname{ssODN}\left(400 \mathrm{ng} / \mu \mathrm{L}\right.$; Ultramer $^{\circledR}$, IDT) were mixed in OptiMEM I (Thermo Fisher Scientific) before electroporation. Fertilized eggs were isolated from the superovulated C57BL/6 J female mice $21 \mathrm{~h}$ after administering human chorionic gonadotropin (hCG). The electroporation was conducted at the 1-cell stage (24-26 h after hCG), and the 2-cell stage embryos were transferred to the oviduct of pseudopregnant ICR females (Charles River Japan, Yokohama, Japan). We confirmed the mutant alleles of the obtained mice (Fig. 1d) by sequencing PCR products.

\section{RT-PCR}

We produced a series of first-strand cDNAs by reversetranscription (RT) from the mice total RNA of eight tissue types (brain, cerebellum, heart, lung, liver, kidney, spleen, and testis) using an oligo-dT primer as previously described $[21,22]$. We used the following primers: Mouse PHY2 exon 3 forward primer: 5'-GTCGTGTCT GAATGGAGTGAGATTATAGAATTCTG-3'; mouse PHY2 exon 5 reverse primer: $5^{\prime}$-ATCCTGGGGTGG CTTCCCTGTATTG-3' (product: 297 bp); mouse glyceraldehyde-3-phosphate dehydrogenase (GAPDH) forward primer: 5'-GCCATCAACGACCCCTTCATTGACCTC3'; mouse GAPDH reverse primer: 5'-GCCATGTAG GCCATGAGGTCCACCAC-3.

\section{In situ hybridization (ISH)}

We performed the ISH as described previously, with minor modifications [23, 24]. C57BL/6 J male mice were anesthetized deeply with a combination of midazolam, medetomidine and butorphanol tartrate and transcardially perfused with $4 \%$ paraformaldehyde (PFA), harvested, post-fixed with $4 \%$ PFA at $4{ }^{\circ} \mathrm{C}$ for $1 \mathrm{~d}$, and equilibrated in $30 \%(\mathrm{w} / \mathrm{v})$ sucrose in phosphate-buffered saline (PBS). Sagittal sections (50 $\mu \mathrm{m}$ thickness) were prepared using a cryostat. All steps were performed at room temperature unless indicated otherwise. Sections were incubated with methanol $(\mathrm{MeOH})$ for $2 \mathrm{~h}$, then washed three times for $10 \mathrm{~min}$ in PBS containing 0.1\% Tween-20 (PBST). Next, they were incubated with $0.5 \mu \mathrm{g} /$ $\mathrm{ml}$ proteinase K (Sigma-Aldrich, St. Louis, MO, USA) in Proteinase $\mathrm{K}$ buffer $(0.1 \mathrm{M}$ Tris $\mathrm{HCl}, \mathrm{pH} 8.0,50 \mathrm{mM}$ EDTA) for $30 \mathrm{~min}$ at $37{ }^{\circ} \mathrm{C}$, followed by incubation with $0.25 \%$ Acetic anhydride in $0.1 \mathrm{M}$ triethanolamine, $\mathrm{pH} 7.0$ for $10 \mathrm{~min}$. Finally, they were washed twice for $5 \mathrm{~min}$ in PBST and incubated with hybridization buffer $(5 \times$ SSC, $50 \%$ formamide, $0.1 \%$ Tween-20, $5 \times$ Denhardt's solution) for $1 \mathrm{~h}$ at $60{ }^{\circ} \mathrm{C}$. Before hybridization, digoxigenin (DIG)-labeled cRNA probes in hybridization buffer were denatured at $80{ }^{\circ} \mathrm{C}$ for $5 \mathrm{~min}$ and then quickly cooled on ice for $10 \mathrm{~min}$. cRNA probes were generated using a DIG RNA labeling kit (Roche, Mannheim, Germany). Hybridization was performed at $60{ }^{\circ} \mathrm{C}$ overnight. Sections were washed in $2 \times$ SSC containing $50 \%$ formamide and $0.1 \%$ Tween-20 (SSCT) twice for $20 \mathrm{~min}$ and incubated with $20 \mu \mathrm{g} / \mathrm{ml}$ RNase (Nippon Gene, Tokyo, Japan) in RNase buffer (10 mM Tris-HCl, pH 8.0, $1 \mathrm{mM}$ EDTA, $0.5 \mathrm{M} \mathrm{NaCl}$ ) for $30 \mathrm{~min}$ at $37^{\circ} \mathrm{C}$. They were then washed twice with $2 \times \mathrm{SSCT}$ for $15 \mathrm{~min}$ at $37^{\circ} \mathrm{C}$ and twice with $0.2 \times \mathrm{SSCT}$ for $15 \mathrm{~min}$ at $37^{\circ} \mathrm{C}$. Then, the sections were incubated with $1 \%$ blocking reagent (Roche; $10 \mathrm{mM}$ maleic acid, $15 \mathrm{mM} \mathrm{NaCl}, \mathrm{pH}$ 7.5) for $1 \mathrm{~h}$, and finally incubated with alkaline phosphatase-conjugated anti-DIG antibody (1:2000, Roche) in blocking reagent at $4{ }^{\circ} \mathrm{C}$ overnight. The sections were washed three times with TNT (0.1 M Tris-HCl, pH 7.5, $0.15 \mathrm{M} \mathrm{NaCl}, 0.05 \%$ Tween-20) for $15 \mathrm{~min}$. For staining with nitroblue tetrazolium chloride/5-bromo-4-chloro-3-indolyl phosphate 4-toluidine salt (NBT/BCIP), the signal was developed in $2 \%(\mathrm{v} / \mathrm{v}) \mathrm{NBT} / \mathrm{BCIP}$ stock solution (Roche) diluted in TNM (0.1 M Tris pH 9.5, $0.1 \mathrm{M} \mathrm{NaCl}, 10 \mathrm{mM} \mathrm{MgCl}$ ) at room temperature overnight. Sections were imaged using a NanoZoomer Digital Pathology virtual slide scanner (Hamamatsu Photonics, Hamamatsu, Japan).

\section{Antibodies}

Rat polyclonal anti-PHY2 antibody was raised against the GST-tagged PHY2 (aa 231-297) that was bacterially expressed, and were affinity-purified against the MBPtagged antigenic proteins that were covalently coupled to CNBr-activated Sepharose 4B.

Rat anti-PHY2 (1 $\mathrm{ng} / \mu \mathrm{l})$, rabbit anti-actin (1:800 dilution; Cat No: A5060; Sigma-Aldrich) mouse 
monoclonal anti-FLAG (1:1,000 dilution; Cat No: F1804; Sigma-Aldrich) and rat monoclonal anti-HA (1:1,000 dilution; Cat No: 1867423; Roche) were used for Western blotting.

The following primary antibodies were used for immunohistochemistry: mouse monoclonal anti-calbindin (1:500 dilution; Cat No: 214011; Synaptic Systems, Goettingen, Germany), mouse monoclonal anti-vGluT2 (1:300 dilution; Cat No: MAB5504; Millipore, Billerica, MA, USA), and rabbit anti-VGAT (1:500 dilution; Cat No: AB5062P; Millipore) antibodies.

\section{Immunohistochemistry}

We performed immunohistochemistry as described previously [25]. C57BL/6 J male mice were anesthetized deeply with a combination of midazolam, medetomidine and butorphanol tartrate and transcardially perfused with PBS and then with Zamboni's fixative (2\% paraformaldehyde in $0.1 \mathrm{M}$ phosphate buffer, $\mathrm{pH} 7.4$, containing $0.2 \%$ picric acid). Tissues were dissected, post-fixed in Zamboni's fixative at $4{ }^{\circ} \mathrm{C}$ for $5 \mathrm{~h}$ and cryoprotected by immersion in $15 \%$ sucrose in PBS overnight at $4{ }^{\circ} \mathrm{C}$. After embedding in Tissue-Tek OCT compound (Sakura Finetek, Tokyo, Japan), tissues were frozen and sectioned at a thickness of $15 \mu \mathrm{m}$ using a cryostat (CM1950, Leica Microsystems, Frankfurt, Germany) at $-18{ }^{\circ} \mathrm{C}$. The sections were air-dried for $1 \mathrm{~h}$ and rinsed in PBS three times. After blocking with $5 \%$ bovine serum albumin (BSA) and 0.3\% Triton X-100 in PBS at room temperature for $1 \mathrm{~h}$, the sections were incubated at $4{ }^{\circ} \mathrm{C}$ overnight with the primary antibodies in immunoreaction buffer $(2 \times$ PBS containing $0.3 \%$ Triton X-100 and 1\% BSA). The sections were then washed in PBS, incubated at room temperature for $1 \mathrm{~h}$ with the appropriate secondary antibodies in immunoreaction buffer, and washed again in PBS. Stained sections were mounted in DAPI Fluoromount$\mathrm{G}^{\circledR}$ mounting medium (SouthernBiotech, Birmingham, AL, USA) and observed under a fluorescence microscope (BX51, Olympus, Tokyo, Japan) equipped with a CCD camera (VB-7000, Keyence, Osaka, Japan).

\section{Rotarod test}

We evaluated the motor control ability of the mice using a rotarod (MK-610A/RKZ; Muromachi Kikai, Tokyo, Japan) as previously described [26, 27]. Briefly, the male mice had to run backwards to maintain their position on top of a rod revolving at $4 \mathrm{rpm}$. The mice were subjected to four trials on the rod accelerating from 4 to $40 \mathrm{rpm}$ in $5 \mathrm{~min}$. There was a $20 \mathrm{~min}$ pause between trials.

\section{Immunoprecipitation}

We performed immunoprecipitation as previously described [28]. Mouse PHY2 cDNA was subcloned in frame in front of the HA epitope tag sequence in pEFBOS plasmid [29] to create the C-terminally HA-tagged PHY2 construct, pEF-BOS-PHY2-HA. Similarly, mouse PAHX, BAI1, and DYRK1A were subcloned in frame in front of the FLAG epitope tag sequence. Transient transfection was performed with lipofectamine 3000 reagent (Thermo Fisher Scientific). Forty-eight hours after transfection, COS-7 cells were harvested and lysed in $1.3 \mathrm{ml}$ of lysis buffer (50 mM HEPES, $\mathrm{pH}$ 7.4, $10 \%$ glycerol, $100 \mathrm{mM} \mathrm{NaCl}, 0.5 \mathrm{mM} \mathrm{MgCl}_{2}, 2 \mathrm{mM}$ EGTA, and 1\% TritonX-100) containing a cocktail of protease inhibitors. After preabsorption with protein A-sepharose, the supernatants were divided equally into two tubes and incubated with $0.5 \mu \mathrm{g}$ normal rat IgG or anti-HA antibody (Cat No: 1867423; Roche), and the immunocomplexes were then associated with protein A-sepharose resins. The resins were washed five times with lysis buffer, and the bound proteins were separated on an SDS-PAGE gel and transferred to a nitrocellulose membrane for analysis with anti-HA or anti-FLAG antibodies.

\section{Abbreviations}

PHYHIPL: Phytanoyl-CoA hydroxylase-interacting protein-like; SNP: Single nucleotide polymorphism; PC: Purkinje cell; CF: Climbing fiber; ML: Molecular layer.

\section{Supplementary Information}

The online version contains supplementary material available at https://doi. org/10.1186/s13041-021-00766-x.

Additional file 1: Fig. S1. Generation of PHY2 Ser19Stop knock-in mice. Alignment of human PHY2 (top) and mouse PHY2 (bottom) protein sequences. Identical amino acids are shown in the middle. The Ser19Stop SNP of human PHY2 registered in the dbSNP (rs7907875) is also shown. The Ser19 is conserved between human and mouse PHY2.

\section{Acknowledgements}

We are grateful to Dr. Nobutake Hosoi (Department of Neurophysiology and Neural Repair, Gunma University Graduate School of Medicine) for help in improving our manuscript.

\section{Authors' contributions}

TS designed the experiments. $\mathrm{HS}, \mathrm{TH}, \mathrm{JNH}, \mathrm{IH}$ and TS performed the experiments. HS, TH, JNH, IH and TS analyzed the data. TH, JNH, YS(Sano) and TS wrote the paper. YS(Shinoda), YI, HH, TF and TS contributed to the discussion. All authors read and approved the final manuscript.

\section{Funding}

This research was supported by Platform Project for Supporting Drug Discovery and Life Science Research (Basis for Supporting Innovative Drug Discovery and Life Science Research (BINDS)) from AMED under Grant Number JP20am0101120 (support number 1783) and also supported by the grants provided by The Ichiro Kanehara Foundation, The Mother and Child Health Foundation, and MEXT/JSPS KAKENHI Grant Numbers JP19K06900.

Availability of data and materials

All data generated or analyzed during this study are included in this published article and its additional information files. 


\section{Declarations}

\section{Ethics approval and consent to participate}

All animal care and treatment procedures followed the Japanese Act on the Welfare and Management of Animals and the Guidelines for the Proper Conduct of Animal Experiments issued by the Science Council of Japan. All animal experiments were approved by the Animal Care and Experimentation Committee of Gunma University and were carried out following the approved guidelines.

\section{Consent for publication}

Not applicable.

\section{Competing interests}

The authors declare that they have no competing interests.

\section{Author details}

1 Education and Research Support Center, Gunma University Graduate School of Medicine, 3-39-22 Showa-machi, Maebashi, Gunma 371-8511, Japan. ${ }^{2}$ Laboratory of Genome Science, Biosignal Genome Resource Center, Institute for Molecular and Cellular Regulation, Gunma University, 3-39-15 Showa-machi, Maebashi 371-8512, Japan. ${ }^{3}$ Department of Applied Biological Science, Faculty of Science and Technology, Tokyo University of Science, 2641 Yamazaki, Noda, Chiba 278-8510, Japan. ${ }^{4}$ Department of Environmental Health, School of Pharmacy, Tokyo University of Pharmacy and Life Sciences, 1432-1 Horinouchi, Hachioji, Tokyo 192-0392, Japan. ${ }^{5}$ Department of Neurophysiology and Neural Repair, Gunma University Graduate School of Medicine, Maebashi, Gunma 371-8511, Japan. ${ }^{6}$ Department of Molecular and Cellular Neurobiology, Gunma University Graduate School of Medicine, 3-39-22 Showa-machi, Maebashi, Gunma 371-8511, Japan. ${ }^{7}$ Center for Translational Neuromedicine, Faculty of Medical and Health Sciences, University of Copenhagen, Blegdamsvej 3B, 2200 Copenhagen N, Denmark.

Received: 18 January 2021 Accepted: 3 March 2021

Published online: 12 March 2021

\section{References}

1. Lee ZH, Kim H, Ahn KY, Seo KH, Kim JK, Bae CS, et al. Identification of a brain specific protein that associates with a refsum disease gene product, phytanoyl-CoA alpha-hydroxylase. Brain Res Mol Brain Res. 2000;75:237-47

2. Koh JT, Lee ZH, Ahn KY, Kim JK, Bae CS, Kim HH, et al. Characterization of mouse brain-specific angiogenesis inhibitor 1 (BAI1) and phytanoyl-CoA alpha-hydroxylase-associated protein 1, a novel BAl1-binding protein. Brain Res Mol Brain Res. 2001;87:223-37.

3. Bescond M, Rahmani Z. Dual-specificity tyrosine-phosphorylated and regulated kinase $1 \mathrm{~A}$ (DYRK1A) interacts with the phytanoyl-CoA alphahydroxylase associated protein 1 (PAHX-AP1), a brain specific protein. Int J Biochem Cell Biol. 2005;37:775-83.

4. Ahn KY, Nam Kl, Kim BY, Cho CW, Jeong SK, Yang KJ, et al. Postnatal expression and distribution of Refsum disease gene associated protein in the rat retina and visual cortex: effect of binocular visual deprivation. Int J Dev Neurosci. 2002:20:93-102.

5. James R, Searcy JL, Le Bihan T, Martin SF, Gliddon CM, Povey J, et al. Proteomic analysis of mitochondria in APOE transgenic mice and in response to an ischemic challenge. J Cereb Blood Flow Metab. 2012;32:164-76.

6. Fu H, Ge B, Chen D, Wu Y, Luo Q, Li X, et al. Phytanoyl-CoA 2-hydroxylaseinteracting protein-like gene is a therapeutic target gene for glioblastoma multiforme. Med Sci Monit. 2019;25:2583-90.

7. Fatemi SH, Aldinger KA, Ashwood P, Bauman ML, Blaha CD, Blatt GJ, et al. Consensus paper: pathological role of the cerebellum in autism. Cerebellum. 2012;11:777-807.

8. Jeong J-W, Tiwari VN, Behen ME, Chugani HT, Chugani DC. In vivo detection of reduced Purkinje cell fibers with diffusion MRI tractography in children with autistic spectrum disorders. Front Hum Neurosci. 2014;8:110.

9. Watanabe M, Kano M. Climbing fiber synapse elimination in cerebellar Purkinje cells. Eur J Neurosci. 2011;34:1697-710.
10. Bravin M, Morando L Vercelli A, Rossi F Strata P Control of spine formation by electrical activity in the adult rat cerebellum. Proc Natl Acad Sci U S A. 1999:96:1704-9.

11. Cesa R, Scelfo B, Strata P. Activity-dependent presynaptic and postsynaptic structural plasticity in the mature cerebellum. J Neurosci. 2007;27:4603-11.

12. Kakizawa S, Miyazaki T, Yanagihara D, lino M, Watanabe M, Kano M. Maintenance of presynaptic function by AMPA receptor-mediated excitatory postsynaptic activity in adult brain. Proc Natl Acad Sci U S A. 2005;102:19180-5.

13. De Zeeuw Cl, Hoebeek FE, Bosman LWJ, Schonewille M, Witter L, Koekkoek SK. Spatiotemporal firing patterns in the cerebellum. Nat Rev Neurosci. 2011:12:327-44.

14. Wulff $P$, Schonewille $M$, Renzi M, Viltono L, Sassoè-Pognetto M, Badura A, et al. Synaptic inhibition of Purkinje cells mediates consolidation of vestibulo-cerebellar motor learning. Nat Neurosci. 2009;12:1042-9.

15. Lazarevic V, Pothula S, Andres-Alonso M, Fejtova A. Molecular mechanisms driving homeostatic plasticity of neurotransmitter release. Front Cell Neurosci. 2013;7:244.

16. De Gois S, Schäfer MKH, Defamie N, Chen C, Ricci A, Weihe E, et al. Homeostatic scaling of vesicular glutamate and GABA transporter expression in rat neocortical circuits. J Neurosci. 2005;25:7121-33.

17. Hulo N, Bairoch A, Bulliard V, Cerutti L, De Castro E, Langendijk-Genevaux PS, et al. The PROSITE database. Nucleic Acids Res. 2006:34:D227-230.

18. Steward A, Adhya S, Clarke J. Sequence conservation in Ig-like domains: the role of highly conserved proline residues in the fibronectin type III superfamily. J Mol Biol. 2002;318:935-40.

19. Sottile J, Mosher DF. N-terminal type I modules required for fibronectin binding to fibroblasts and to fibronectin's III1 module. Biochem J. 1997;323(Pt 1):51-60.

20. Horii T, Morita S, Kimura M, Terawaki N, Shibutani M, Hatada I. Efficient generation of conditional knockout mice via sequential introduction of lox sites. Sci Rep. 2017;7:7891.

21. Sadakata T, Mizoguchi A, Sato Y, Katoh-Semba R, Fukuda M, Mikoshiba K, et al. The secretory granule-associated protein CAPS2 regulates neurotrophin release and cell survival. J Neurosci. 2004;24:43-52.

22. Sadakata T, Washida M, Furuichi T. Alternative splicing variations in mouse CAPS2: differential expression and functional properties of splicing variants. BMC Neurosci. 2007;8:25.

23. Sadakata T, Kakegawa W, Mizoguchi A, Washida M, Katoh-Semba R, Shutoh F, et al. Impaired cerebellar development and function in mice lacking CAPS2, a protein involved in neurotrophin release. J Neurosci. 2007;27:2472-82

24. Sano Y, Shobe JL, Zhou M, Huang S, Shuman T, Cai DJ, et al. CREB regulates memory allocation in the insular cortex. Curr Biol. 2014;24:2833-7.

25. Hosoi N, Shibasaki K, Hosono M, Konno A, Shinoda Y, Kiyonari H, et al. Deletion of class II ADP-ribosylation factors in mice causes tremor by the Nav1.6 loss in cerebellar Purkinje cell axon initial segments. J Neurosci. 2019:39:6339-53.

26. Sadakata T, Shinoda Y, Oka M, Sekine Y, Sato Y, Saruta C, et al. Reduced axonal localization of a Caps2 splice variant impairs axonal release of BDNF and causes autistic-like behavior in mice. Proc Natl Acad Sci U S A. 2012:109:21104-9.

27. Watanave M, Matsuzaki Y, Nakajima Y, Ozawa A, Yamada M, Hirai H. Contribution of thyrotropin-releasing hormone to cerebellar long-term depression and motor learning. Front Cell Neurosci. 2018;12:490.

28. Sadakata T, Washida M, Iwayama Y, Shoji S, Sato Y, Ohkura T, et al. Autisticlike phenotypes in Cadps2-knockout mice and aberrant CADPS2 splicing in autistic patients. J Clin Invest. 2007;117:931-43.

29. Mizushima S, Nagata S. pEF-BOS, a powerful mammalian expression vector. Nucleic Acids Res. 1990;18:5322.

\section{Publisher's Note}

Springer Nature remains neutral with regard to jurisdictional claims in published maps and institutional affiliations. 